\title{
CONFORMAL DEFORMATIONS OF THE SMALLEST EIGENVALUE OF THE RICCI TENSOR
}

\author{
PENGFEI GUAN AND GUOFANG WANG
}

\begin{abstract}
We consider deformations of metrics in a given conformal class such that the smallest eigenvalue of the Ricci tensor to be a constant. It is related to the notion of minimal volumes in comparison geometry. Such a metric with the smallest eigenvalue of the Ricci tensor to be a constant is an extremal metric of volume in a suitable sense in the conformal class. The problem is reduced to solve a Pucci type equation with respect to the Schouten tensor. We establish a local gradient estimate for this type of conformally invariant fully nonlinear uniform elliptic equations. Combining it with the theory of fully nonlinear equations, we establish the existence of solutions for this equation.
\end{abstract}

\section{INTRODUCTION}

The Ricci curvature tensor of a Riemannian metric plays an important role in comparison geometry for Riemannian manifolds, in particular the lower bounds of Ricci curvature. See for instance [2, 13, 30. In this paper, we are interested in conformal deformations of the smallest eigenvalue of the Ricci tensor. Let $\left(M^{n}, g_{0}\right)$ be an $n$-dimensional compact Riemannian manifold and $\left[g_{0}\right]$ its conformal class. And let $R i c_{g}$ and $R_{g}$ be the Ricci curvature tenser and the scalar tensor of a metric $g$ respectively. Define $\min \operatorname{Ric}_{g}(x)$ the smallest eigenvalue of $g^{-1} \cdot R i c_{g}$ at $x \in M$. Our problem is to find a conformal metric $g=e^{-2 u} g_{0}$ such that

$$
\min \operatorname{Ric}_{g}(x)=\text { constant. }
$$

It turns out that the problem is equivalent to solving an interesting fully nonlinear uniform elliptic equation. First we recall that the Schouten tensor of the metric $g$ is defined by

$$
S_{g}=\frac{1}{n-2}\left(R i c_{g}-\frac{R_{g}}{2(n-1)} g\right) .
$$

Let $\Lambda=\left(\lambda_{1}, \lambda_{2}, \cdots, \lambda_{n}\right) \in \mathbb{R}^{n}$. Assume that $\lambda_{1} \leq \lambda_{2} \leq \cdots \leq \lambda_{n}$. For an integer $1 \leq p \leq n-1$, define a function $G_{p}: \mathbb{R}^{n} \rightarrow \mathbb{R}$ by

$$
G_{p}(\Lambda)=(n-p) \sum_{i \leq p} \lambda_{i}+p \sum_{i>p} \lambda_{i}
$$

For a symmetric matrix $A, G_{p}(A)=G_{p}(\Lambda)$, where $\Lambda$ is the set of eigenvalues of $A$. It is easy to check $\min$ Ric $=G_{1}\left(g^{-1} \cdot S_{g}\right)$.

2000 Mathematics Subject Classification. Primary 53C21; Secondary 35J60, 58E11 .

Key words and phrases. fully nonlinear equation, local estimates, conformal deformation, Ricci tensor, minimal volume.

Research of the first author was supported in part by an NSERC Discovery Grant. 
We may also ask if there is a conformal metric with a constant $W_{p}(g):=G_{p}\left(g^{-1} \cdot S_{g}\right) . W_{p}(g)$ is also an interesting geometric object, which will be called $p$-Weitzenböck curvature, for it arises from the Weitzenböck formula for $p$-forms in a locally conformally flat manifold. See the Appendix. We will consider the following general equation

$$
W_{p}(g)(x)=f(x), \quad \forall x \in M .
$$

We first treat the case when the background metric has negative curvature, i.e., $W_{p}\left(g_{0}\right)<0$.

Theorem 1. Let $\left(M, g_{0}\right)$ be a compact Riemannian manifold and $1 \leq p \leq n$. Suppose that $W_{p}\left(g_{0}\right)(x)<0$ for any $x \in M$, then there is a unique $C^{2, \alpha}$ metric $g \in\left[g_{0}\right]$ for some $\alpha>0$ such that $W_{p}(g)(x)=-1, \quad \forall x \in M$.

A geometric consequence of Theorem 1 is the existence of an extremal metric in the conformal class with minimal volume. Although (2) has no variational structure in general, a solution of (2) does achieve the minimum of the minimal volume in a conformal class in this case. The following is a simple consequence, see section 4 for other related results.

Corollary 1. Suppose that $\min \operatorname{Ric}_{g_{0}}(x)<0$ for any $x \in M$. Then there is a unique conformal metric $g^{*} \in\left[g_{0}\right]$ such that $\operatorname{vol}\left(g^{*}\right)=\min \operatorname{vol}(g)$, where minimum is taken over all $g \in\left[g_{0}\right]$ with $\min \operatorname{Ric}_{g}(x) \geq-1$. The extremal metric $g^{*}$ is characterized by a unique solution to equation $\min \operatorname{Ric}_{g^{*}}(x)=-1, \forall x \in M$.

This Corollary is related to the minimal volumes considered by Gromov [12] and LeBrun [23]

We now turn to the case of the positive Ricci curvature.

Theorem 2. Let $\left(M, g_{0}\right)$ be a compact Riemannian manifold with Ric g $_{0}>0$. Then there is a conformal metric $g \in\left[g_{0}\right], g \in C^{2, \alpha}(M)$ for some $\alpha>0$ such that $\min$ Ric $_{g}(x)=n-1$ for all $x \in M$.

The positivity of $p$-Weitzenböck curvature for $1<p \leq n / 2$ plays an important role in the investigation of the topological structure of locally conformally flat manifolds in [14] and the Appendix.

Theorem 3. Let $\left(M, g_{0}\right)$ be an $n$-dimensional smooth compact locally conformally flat manifold with $W_{p}\left(g_{0}\right)>0$ and $p \leq n / 2$. If $\left(M, g_{0}\right)$ is not conformally equivalent to the standard $n$-sphere, then there exists $g \in\left[g_{0}\right], g \in C^{2, \alpha}(M)$ for some $\alpha>0$ such that

$$
W_{p}(g)(x)=1, \quad \forall x \in M \text {. }
$$

Furthermore, the solution space is compact. That is, there is positive constant $C>0$, such that

$$
\|u\|_{C^{2, \alpha}} \leq C
$$

for any $C^{2, \alpha}$ solution $g=e^{-2 u} g_{0}$ of (3).

One of the interesting aspect of this paper is that our geometric problem is deduced to a Pucci type fully nonlinear uniformly elliptic equations with respect to the Schouten tensor. These equations have been studied extensively in Euclidean domains, in particular in connection to stochastic optimization. With the breakthrough of the Krylov-Safonov's Harnack estimate 
22 for non-divergent elliptic equations, it followed the fundamental Evans-Krylov theorem [10. 21] on the Hölder regularity of the second derivatives. The deep results of Caffarelli [5] and Safonov 33, 34 provide further improvement of such Hölder estimates bypassing $C^{2}$ estimates. Caffarelli's perturbation theory is very important for our application in conformal geometry. Especially some of our geometric equations are in terms of convex operators, where $C^{2}$ estimates are difficult to obtain with standard approaches. The core of Caffarelli's perturbation theory is the $C^{2}$ estimates for simple model equations, which were established by him for the concave and convex operators. To apply the Caffarelli perturbation theory for our equations, we need one more crucial estimate, that is a local gradient bound depending only on one side bound of solutions. As in the case for nonlinear equations in conformal geometry, this is a key step for the blow-up analysis. The Bishop-Gromov volume comparison theorem also pays an important role in the proof of Theorem 3 .

The paper is organized as follows. In Section 2, we discuss briefly our uniformly elliptic fully nonlinear equations and Caffarelli's results. We prove the local gradient estimates in Section 3. In Section 4 we discuss the case of negative curvature and the minimal volume in a conformal class. We prove Theorem 2 and Theorem 3 in Section 5.

Acknowledgement. We would like to thank Claude LeBrun for helpful conversations with the first author at AIM's workshop on "Conformal Structure in Geometry" in Palo Alto. When informed our results on the conformal deformations of the smallest eigenvalue of Ricci tensor, he pointed to us a possible application to the minimal volumes. Corollary 1 was inspired by conversations with him.

\section{Fully nONLINEAR UNIFORMLY ELliptiC EQUATIONS AND CAFFARELli's estimates}

In this section, we will deduce our problem to fully nonlinear uniformly elliptic elliptic equations. We will make use of Caffarelli's fundamental $W^{2, p}$ and $C^{2, \alpha}$ estimates in [5] (see also Safonov's work on $C^{2, \alpha}$ estimates in [34]). Some of these results have been subsequently generalized to certain type of equations of form $F\left(\nabla^{2} u, \nabla u, x\right)=f(x)$ by L. Wang in [39] as well as for the case of parabolic equations. As we will see that equation (2) involves $\nabla u$ in a delicate way, we need certain appropriate a priori estimates depending only on one side bound of $u$ (to be more explicit, $e^{-2 \inf u}$ ). This type of local gradient estimates will be established in the next section.

Let $\left(M^{n}, g_{0}\right)$ be a compact, oriented Riemannian manifold of dimension $n>2$. Let $\left[g_{0}\right]$ be the conformal class of $g_{0}$. For any $g \in\left[g_{0}\right]$, we denote $R i c_{g}, S_{g}$ the Ricci tensor and the Schouten tensor of the metric $g$ respectively. We write

$$
\begin{aligned}
& \mathcal{R}_{g}^{s}(x)=\text { smallest eigenvalue of } g^{-1} \operatorname{Ric}_{g}(x), \\
& \mathcal{R}_{g}^{l}(x)=\text { largest eigenvalue of } g^{-1} \operatorname{Ric}_{g}(x), \\
& \mathcal{S}_{g}^{s}(x)=\text { smallest eigenvalue of } g^{-1} S_{g}(x), \\
& \mathcal{S}_{g}^{l}(x)=\text { largest eigenvalue of } g^{-1} S_{g}(x) .
\end{aligned}
$$


It is clear that $\mathcal{R}_{g}^{s}$ and $\mathcal{R}_{g}^{l}$ do not depend on the choice of coordinates. From the definition of the Schouten tensor

$$
S_{g}=\frac{1}{n-2}\left(R i c_{g}-\frac{R_{g}}{2(n-1)}\right)
$$

we have the following relations

$$
\begin{aligned}
& \mathcal{R}_{g}^{s}(x)=(n-2) \mathcal{S}_{g}^{s}(x)+\operatorname{tr}\left(S_{g}(x)\right), \\
& \mathcal{R}_{g}^{l}(x)=(n-2) \mathcal{S}_{g}^{l}(x)+\operatorname{tr}\left(S_{g}(x)\right) .
\end{aligned}
$$

If $g=e^{-2 u} g_{0}$, there is a transformation formula between two Schouten tensors

$$
S_{g}=\nabla^{2} u+\nabla u \otimes \nabla u-\frac{|\nabla u|^{2} g_{0}}{2}+S_{g_{0}}
$$

where all covariant derivatives are with respect to $g_{0}$. (The same convention will be used in the rest of this paper, unless it is stated otherwise). Locally, denoting $S_{i j}$ the Schouten tensor of $g_{0}$ under the frame, we have

$$
\left(S_{g}\right)_{i j}=u_{i j}+u_{i} u_{j}-\frac{|\nabla u|^{2}}{2} \delta_{i j}+S_{i j}
$$

For any symmetric matrix $A$, we denote $\lambda_{s}(A)$ and $\lambda_{l}(A)$ to be the smallest and largest eigenvalues of $A$ respectively. And we denote $\sigma_{1}(A)=\operatorname{tr}(A)$. We obtain the equations for constant $\mathcal{R}_{g}^{s}$ and $\mathcal{R}_{g}^{l}$ respectively:

$$
\begin{aligned}
& F_{*}(u)=:\left((n-2) \lambda_{s}+\sigma_{1}\right)\left(\nabla^{2} u+d u \otimes d u-\frac{|\nabla u|^{2}}{2} g_{0}+S_{g_{0}}\right)=e^{-2 u} \mathcal{R}_{g}^{s}, \\
& F^{*}(u)=:\left((n-2) \lambda_{l}+\sigma_{1}\right)\left(\nabla^{2} u+d u \otimes d u-\frac{|\nabla u|^{2}}{2} g_{0}+S_{g_{0}}\right)=e^{-2 u} \mathcal{R}_{g}^{l} .
\end{aligned}
$$

Hence $F_{*}$ and $F^{*}$ are uniformly elliptic with ellipticity constants 1 and $n-1$. It is clear that $F_{*}$ is concave and $F^{*}$ is convex. We also note that $F^{*}$ and $F_{*}$ are homogeneous of degree 1.

There are other similar fully nonlinear equations arising in the Weitzenböck formula for $p$-form on local conformally flat manifolds. Let $\Lambda=\left(\lambda_{1}, \lambda_{2}, \cdots, \lambda_{n}\right) \in \mathbb{R}^{n}$ be the set of eigenvalues of a symmetric matrix $A$. For an integer $1 \leq p \leq n / 2$, define a function $G_{p}: \mathbb{R}^{n} \rightarrow \mathbb{R}$ by

$$
G_{p}(A)=G_{p}(\Lambda)=\min \left\{(n-p) \sum_{k \leq p} \lambda_{i_{k}}+p \sum_{k>p} \lambda_{i_{k}}\right\}
$$

where min is over all permutations of $1,2, \cdots, n$. We define $W_{p}(g)$ the $p$-Weitzenböck curvature of $g$ by

$$
W_{p}(g)=G_{p}\left(g^{-1} \cdot S_{g}\right)
$$

It is easy to check $\mathcal{R}_{g}^{s}=W_{1}(g)$. The $p$-Weitzenböck curvature is as much interest as the scalar curvture, at least for locally conformally flat manifold. For example, from the Weitzenböck formula one can easily show that a locally conformally flat manifold with positive $p$-Weitzenböck curvature has vanishing $q$-cohomology group for $n / 2-p \leq q \leq n / 2+p$ (for $p \leq n / 2$ ). 
We may also ask whether we can find a conformal metric with constant $W_{p}$. The corresponding equation is

$$
G_{p}\left(\nabla^{2} u+d u \otimes d u-\frac{|\nabla u|^{2}}{2} g_{0}+S_{g_{0}}\right)=\text { constant } \cdot e^{-2 u} .
$$

Equation (9) is also uniformly elliptic. It is easy to see that (9) is concave for $p \leq \frac{n}{2}$. From the transformation formula (6), one can check that Equation (9) is also conformally invariant. Hence, in general there is no compactness for equation (9).

We also define for $p \geq n / 2$ a function $G_{p}: \mathbb{R}^{n} \rightarrow \mathbb{R}$ by

$$
G_{p}(A)=G_{p}(\Lambda)=\max \left\{(n-p) \sum_{k \leq p} \lambda_{i_{k}}+p \sum_{k>p} \lambda_{i_{k}}\right\}
$$

where max is over all permutations of $1,2, \cdots, n$. We still define $W_{p}(g)=G_{p}\left(g^{-1} \cdot S_{g}\right)$. It is clear that $\mathcal{R}_{g}^{l}=W_{n-1}(g)$. We can also consider equation (9) for $p \geq n / 2$. For $p \geq n / 2$ equation (19) is still uniformly elliptic, but it is convex. Since $G_{p}$ is homogeneous of order 1 , we may rewrite equation (2) as the following equivalent equation by setting $v=e^{u}$

$$
G_{p}\left(\nabla^{2} v+S_{g_{0}} v\right)=p(n-p) \frac{|\nabla v|^{2}}{v}+\frac{f}{v} .
$$

The function $G_{p}$ is only Lipschitz, when $p \neq n / 2$. (Remark that when $p=n / 2$, equation (9) is equivalent to the Yamabe equation.) One can find a sequence of smooth functions $\left\{F_{k}\right\}$ such that $F_{k}$ uniformly converges to $G_{p}$ in any compact domain of $\mathbb{R}^{n}$ and homogeneous 1 outside the unit ball in $\mathbb{R}^{n}$, i.e., $F_{k}(x)=|x| F\left(\frac{x}{|x|}\right)$ for $|x| \geq 1$. Furthermore, for $p<n / 2(p>n / 2) F_{k}$ is concave (convex).

One may consider a more general class of conformal equations. Let $\mathcal{S}$ be the space of symmetric 2-tensors on $M$. Let $F: \mathcal{S} \rightarrow \mathbb{R}$ a real continuous function. We consider the following general equation

$$
F\left(e^{-2 u}\left(\nabla^{2} u+d u \otimes d u-\frac{|\nabla u|^{2}}{2} g_{0}+S_{g_{0}}\right)\right)=f(x),
$$

for some function $f: M \rightarrow \mathbb{R}$. $F$ is uniformly elliptic with constants $\lambda_{0}$ and $\Lambda_{0}$ if there exists two positive constants $\lambda_{0}$ and $\Lambda_{0}$ such that for any $W \in \mathcal{S}$

$$
\lambda_{0}\|N\| \leq F(W+N)-F(W) \leq \Lambda_{0}\|N\| \quad \forall N \geq 0,
$$

here by $N \geq 0$ means that $N$ is nonnegative definite and $\|N\|=\sup _{|v|=1}|N v|$. If $F$ is uniformly elliptic, we call equation (11) a uniformly elliptic fully nonlinear equation with ellipticity constants $\lambda_{0}$ and $\Lambda_{0}$. There are many typical uniformly elliptic fully nonlinear equations. Our equation (9) is similar to the Pucci equation, see [5]. Let $\mathcal{M}^{ \pm}$be the Pucci's extremal operators, namely for two given constant $0<\lambda_{0}<\Lambda_{0}$ and $W \in \mathcal{S}$

$$
\begin{aligned}
\mathcal{M}^{-}(W) & =\lambda_{0} \sum_{e_{i}>0} e_{i}+\Lambda_{0} \sum_{e_{i}<0} e_{i}, \\
\mathcal{M}^{+}(W) & =\Lambda_{0} \sum_{e_{i}>0} e_{i}+\lambda_{0} \sum_{e_{i}<0} e_{i},
\end{aligned}
$$


where $e_{i}=e_{i}(W)$ are the eigenvalues of $W$. One can also consider

$$
\begin{aligned}
& \mathcal{M}^{-}\left(e^{-2 u}\left(\nabla^{2} u+d u \otimes d u-\frac{|\nabla u|^{2}}{2} g_{0}+S_{g_{0}}\right)\right)=1, \\
& \mathcal{M}^{+}\left(e^{-2 u}\left(\nabla^{2} u+d u \otimes d u-\frac{|\nabla u|^{2}}{2} g_{0}+S_{g_{0}}\right)\right)=1 .
\end{aligned}
$$

$\mathcal{M}^{-}$is concave, while $\mathcal{M}^{+}$is convex.

We now deduce $C^{2, \alpha}$ estimates for equation (2) from the work of Caffarelli [5] assuming the gradient bound and a lower bound of $u$. The crucial part in Caffarelli's perturbation theory for uniformly elliptic fully nonlinear equation $F\left(\nabla^{2} u, x\right)=f(x)$ is the $C^{1,1}$ interior estimates for $F\left(\nabla^{2} u, x_{0}\right)=$ constant. He obtained such fundamental estimates for concave or convex operator $F$ (note that here concavity and convexity of $F$ can always be switched by $\tilde{F}(\Lambda)=-F(-\Lambda)$ ). Though Caffarelli proved these estimates in [5] for equations with flat metric, his arguments work under general Riemannian metrics. And the generalization of Caffarelli's estimates by L. Wang [39] to uniformly elliptic equations of form $F\left(\nabla^{2} u, \nabla u, u, x\right)$ gives the following $C^{2, \alpha}$ estimates for equations of type (9).

Theorem 4 ( $C^{2, \alpha}$-estimates). Suppose $F$ is a uniformly elliptic concave operator with elliptic constants $\lambda_{0}, \Lambda_{0}$. Let $B_{1}$ be a unit disk in a compact Riemannian manifold $M$ and $f, h \in C^{1}\left(B_{1}\right)$. Suppose $g=e^{-2 u} g_{0}$ with $|\nabla u|_{B_{1}} \leq A$ is a solution of equation

$$
F\left(\nabla^{2} u+d u \otimes d u-\frac{|\nabla u|^{2}}{2} g_{0}+S_{g_{0}}\right)=e^{-2 u} f(x), \quad x \in B_{1},
$$

then there exist $\alpha>0$ and $C>0$ depending only on $\lambda_{0}, \Lambda_{0}, A,\|u\|_{C^{0}\left(B_{1}\right)}$ and $g_{0}$ such that

$$
\|u\|_{C^{2, \alpha}\left(B_{\frac{1}{2}}\right)} \leq C \text {. }
$$

In fact, we may directly apply Caffarelli's estimates [5] to obtain $C^{2, \alpha}$ estimates for equations of type (10). Let $B_{1}$ be a unit disk in a compact Riemannian manifold $M$ and $f, h \in C^{1}\left(B_{1}\right)$. Suppose $g=e^{-2 u} g_{0}=v^{-2} g_{0}$ with $\left|\frac{\nabla v}{v}\right|_{B_{1}} \leq A$ is a solution of equation

$$
F\left(\nabla^{2} v(x)+v(x) S_{g_{0}}(x)\right)=h(x) \frac{|\nabla v(x)|^{2}}{v(x)}+\frac{f(x)}{v(x)}, \quad x \in B_{1} .
$$

Since $F$ is concave, by Theorem 6.6 in [6], the equation

$$
F\left(\nabla^{2} v+S_{g_{0}}\left(x_{0}\right) v\right)=\text { constant }
$$

has $C^{1,1}$ interior estimates for any $x_{0} \in B_{1}$. It follows from Theorem $7.1 \mathrm{in}[$ ] that equation (15) has interior $W^{2, p}$ estimate for any $n<p<\infty$ since $\frac{|\nabla v|}{v} \leq A$. This in turn gives $C^{1, \beta}$ a priori bound for the solution $v$ of equation (15) for all $0<\beta<1$. Finally estimate (14) for $v=e^{u}$ follows from Theorem 8.1 in [6] since the right hand side of equation (15) is a $C^{\beta}$ function now.

\section{LocAl GRADIEnt ESTimates}

In this section, we establish a local gradient estimates for solutions $g=e^{-2 u} g_{0}$ of equation (21) in the case $p \leq n / 2$, depending only on lower bound of $u$. 
Theorem 5 (Local gradient estimates). Suppose $F$ is concave and uniformly elliptic with ellipticity constants $\lambda_{0}, \Lambda_{0}$. Let $B_{1}$ be a unit disk in a compact Riemannian manifold $M$ and u a $C^{2}$ solution of the following equation

$$
F\left(\nabla^{2} u+d u \otimes d u-\frac{|\nabla u|^{2}}{2} g_{0}+S_{g_{0}}\right)=e^{-2 u} f(x), \quad x \in B_{1}
$$

for a $C^{1}$ function $f: B_{1} \rightarrow \mathbb{R}$. Then there is a constant $C>0$ depending only on $\lambda_{0}, \Lambda_{0}, g_{0}$ such that

$$
|\nabla u|^{2}(x) \leq C\left(1+\|f\|_{C^{1}\left(B_{1}\right)} e^{-2 \inf _{B_{1}} u}\right), \quad \text { for any } x \in B_{1 / 2} .
$$

Combining Theorem 5 and Theorem 4 , we deduce the following.

Corollary 2 (Local $C^{2, \alpha}$-estimates). Let $B_{1}$ be a unit disk in a compact Riemannian manifold $M$ and $f \in C^{1}\left(B_{1}\right)$. Suppose $u$ is a solution of equation (16), then there is a constant $C>0$ depending only on $\lambda_{0}, \Lambda_{0}, g_{0},\|f\|_{C^{1} B_{1}}, \inf _{B_{1}} u$, such that

$$
\|u\|_{C^{2, \alpha}\left(B_{\frac{1}{2}}\right)} \leq C \text {. }
$$

In particular, (18) is true for any solution of (2) when $p \leq \frac{n}{2}$.

We note that Corollary 2 guarantees that we can use the so-called blow-up analysis to study the fully nonlinear equation (2). We remark that the local gradient estimates (17) does not true for $p=n-1$. The local gradient estimates were first obtained for $\sigma_{k}$ conformal invariant equations in [15] and for $\frac{\sigma_{k}}{\sigma_{l}}$ conformal invariant equations in [17], though in general fully nonlinear equations have no local estimates. The operator $G_{p}$ we are considering here is only Lipschitz, which we will deal with by a smoothing argument. Actually we can prove the local gradient estimates for a more general class of uniformly elliptic fully nonlinear conformal equations.

We first prove the local gradient estimates for $C^{2}$ uniformly elliptic operator $F$. Let $F: \mathbb{R}^{n} \rightarrow$ $\mathbb{R}$ is a $C^{2}$ symmetric function and consider the following equation

$$
F\left(\nabla^{2} u+d u \otimes d u-\frac{|\nabla u|^{2}}{2} g_{0}+S_{g_{0}}\right)=\tilde{f}
$$

for some $C^{1}$ function $\tilde{f}$. We denote the left hand side of (19) by $F(W)$ and set

$$
F^{i j}=\frac{\partial F}{\partial w_{i j}}
$$

where $w_{i j}$ is the entry of the matrix $W$. As mentioned above, $F(W)=F(\Lambda)$, where $\Lambda$ is the set of eigenvalues of $W$.

Proposition 1. Let $B_{1}$ be a unit disk in a compact Riemannian manifold $M$ and $u$ a $C^{3}$ solution of (19) for a $C^{1}$ function $\tilde{f}: B_{1} \rightarrow \mathbb{R}$. Let $F: \mathcal{S} \rightarrow \mathbb{R}$ be a $C^{2}$ function satisfying

(1) $F$ is an uniformly elliptic with ellipticity constants $\lambda_{0}$ and $\Lambda_{0}$

(2) $F$ is concave.

Then for any $\rho \in C_{0}^{2}\left(B_{1}\right)$ with $0 \leq \rho(x) \leq 1$, there is a constant $C>0$ depending only on $\lambda_{0}$, $\Lambda_{0},\|\rho\|_{C}^{2}\left(B_{1}\right) \|$ and $g_{0}$ such that

$$
\max _{B_{1}}\left\{\rho(x)|\nabla u|^{2}(x)\right\} \leq C\left(1+\max _{B_{1}}\{\rho(x)|\nabla \tilde{f}(x)|\}\right) .
$$


Proof of Proposition [1. The Proof follows closely the argument given in [15] and [16]. As in [15], we first reduce the proof of the Lemma to the following claim.

Claim. There is a constant $A_{0}$ depending, such that

$$
\sum_{i, j} F^{i i} \tilde{u}_{i j}^{2} \geq A_{0}^{-\frac{5}{8}} \sum_{i} F^{i i}|\nabla u|^{4},
$$

where $\tilde{u}_{i j}=u_{i j}+S_{i j}$.

For convenience of the reader, we sketch the reduction. Let $\rho$ be a test function $\rho \in C_{0}^{2}\left(B_{1}\right)$. We may assume

$$
|\nabla \rho(x)| \leq 2 b_{0} \rho^{1 / 2}(x) \quad \text { and } \quad\left|\nabla^{2} \rho\right| \leq b_{0}, \text { in } B_{1},
$$

for $b_{0}>1$. Set $H=\rho|\nabla u|^{2}$. Our aim is to bound $\max _{B_{1}} H$. Let $x_{0} \in B_{1}$ be a maximum point of $H$ and assume that $W$ is a diagonal matrix at the point $x_{0}$ by choosing a suitable normal coordinates around $x_{0}$. Set $\lambda_{i}=w_{i i}$ and $\Lambda=\left(\lambda_{1}, \lambda_{2}, \cdots, \lambda_{n}\right)$. Since $W$ is diagonal at $x_{0}$, we have at $x_{0}$

$$
w_{i i}=u_{i i}+u_{i}^{2}-\frac{1}{2}|\nabla u|^{2}+S_{i i}, \quad u_{i j}=-u_{i} u_{j}-S_{i j}, \quad \forall i \neq j,
$$

where $S_{i j}$ are entries of $S_{g_{0}}$. We may assume that

$$
H\left(x_{0}\right) \geq b_{0}^{2} A_{0}^{2},
$$

for some large, but fixed constant $A_{0}>0$ which will be fixed later. We may also assume that

$$
\left|S_{g_{0}}\right|\left(x_{0}\right) \leq A_{0}^{-1}|\nabla u|^{2}\left(x_{0}\right) .
$$

Otherwise, we are done. The fact that the derivatives of $H$ at $x_{0}$ vanish imply

$$
\left|\sum_{l=1}^{n} u_{i l} u_{l}\right|\left(x_{0}\right) \leq \frac{|\nabla u|^{3}}{A_{0}}\left(x_{0}\right) \quad \text { for any } i \text {. }
$$

Applying the maximum principle to $H$, we have

$$
0 \geq F^{i j} H_{i j}=F^{i j}\left\{\left(-2 \frac{\rho_{i} \rho_{j}}{\rho}+\rho_{i j}\right)|\nabla u|^{2}+2 \rho u_{l i j} u_{l}+2 \rho u_{i l} u_{j l}\right\} .
$$

The first term in the left hand side of (26) is bounded from below by $-10 n b_{0} \Lambda_{0}|\nabla u|^{2}$. By using equation (19) and inequality (25), the second term can be bounded by

$$
\begin{aligned}
\sum_{i, j, l} F^{i j} u_{i j l} u_{l} & \geq \sum_{l} F_{l} u_{l}-2 \sum_{i, l} F^{i i} u_{i l} u_{l} u_{i}+\sum_{i, l} F^{i i} u_{j l} u_{j} u_{l}-C|\nabla u|^{2} \sum_{i} F^{i i} \\
& \geq-|\tilde{f}|^{2}-2 n \sum_{i} F^{i i} \frac{|\nabla u|^{4}}{A_{0}}-C|\nabla u|^{2},
\end{aligned}
$$


where $C>0$ depends only on $g_{0}$ and $\Lambda_{0}$. See also (2.20) in [15]. It is easy to see that the third term is bounded by the Claim. Hence if the Claim is true, from (26) we have

$$
0 \geq-C|\nabla u|^{2}-\rho|\nabla \tilde{f}|^{2}-\rho \sum_{i} F^{i i} \frac{|\nabla u|^{4}}{A_{0}}+\rho A_{0}^{-\frac{5}{8}} \sum_{i} F^{i i}|\nabla u|^{4} .
$$

Multiplying (28) by $\rho$, we have

$$
0 \leq \sum_{i} F^{i i}\left(A_{0}^{-\frac{5}{8}}-A_{0}^{-1}\right) H^{2}-C H-\rho^{2}|\nabla \tilde{f}|^{2}
$$

from which we have (20).

Now we prove the Claim. By (23), we have

$$
\begin{aligned}
\sum_{i, l} F^{i i} \tilde{u}_{i l}^{2} & =\sum_{i} F^{i i} \tilde{u}_{i i}^{2}+\sum_{i \neq l} F^{i i} u_{i}^{2} u_{l}^{2} \\
& =\sum_{i} F^{i i}\left\{\tilde{u}_{i i}^{2}+u_{i}^{2}\left(|\nabla u|^{2}-u_{i}^{2}\right)\right\} \\
& =\sum_{i} F^{i i}\left(w_{i i}^{2}-2 u_{i}^{2} w_{i i}+w_{i i}|\nabla u|^{2}+\frac{|\nabla u|^{4}}{4}\right) .
\end{aligned}
$$

Set $\delta_{0}=A_{0}^{-1 / 4}<0.1$. We divide the set $I=\{1,2, \cdots, n\}$ as in [15] into two parts:

$$
I_{1}=\left\{\left.i \in I\left|u_{i}^{2} \geq \delta_{0}\right| \nabla u\right|^{2}\right\} \quad \text { and } \quad I_{2}=\left\{\left.i \in I\left|u_{i}^{2}<\delta_{0}\right| \nabla u\right|^{2}\right\} .
$$

It is clear that $I_{1}$ is non-empty.

Case 1. There is $j_{0}$ satisfying

$$
\tilde{u}_{j j}^{2} \leq \delta_{0}^{2}|\nabla u|^{4} \quad \text { and } \quad u_{j}^{2}<\delta_{0}|\nabla u|^{2} .
$$

We may assume that $j_{0}=n$. We have $\left|w_{n n}+\frac{|\nabla u|^{2}}{2}\right|=\left|\tilde{u}_{n n}+u_{n}^{2}\right|<2 \delta_{0}|\nabla u|^{2}$ by (30). From (25) and (23), we have

$$
\left|w_{i i}-\frac{|\nabla u|^{2}}{2}\right|=\left.\left|u_{i i}+u_{i}^{2}-\right| \nabla u\right|^{2}+\left.S_{i i}\left|\leq 3 \delta_{0}^{2}\right| \nabla u\right|^{2}
$$

for any $i \in I_{1}$.

Using these estimates, we repeat the derivation of equation (2.38) in [15] to obtain

$$
\sum_{i, l} F^{i i} \tilde{u}_{i l}^{2} \geq \tilde{F}^{1} \frac{|\nabla u|^{4}}{4}-\tilde{F}^{1}|\nabla u|^{4}+F^{n n} \frac{|\nabla u|^{4}}{4}+\left(1-32 \delta_{0}^{2}\right) \frac{|\nabla u|^{4}}{4} \sum_{i} F^{i i}
$$

where $\tilde{F}^{1}:=\max _{i \in I_{1}} F^{i i}$. Recall that $I_{1}$ is necessarily non-empty. We assume $1 \in I_{1}$ with $F^{11}=\tilde{F}^{1}$. The concavity of $F$ implies that

$$
F^{n n} \geq F^{11}
$$


for $w_{11}>w_{n n}$. Hence, from (31) we have

$$
\begin{aligned}
\sum_{i, l} F^{i i} \tilde{u}_{i l}^{2} & \geq-16 \delta_{0}^{2}|\nabla u|^{4}+\left(1-32 \delta_{0}^{2}\right) \frac{|\nabla u|^{4}}{4} \sum_{i=2}^{n-1} F^{i i} \\
& \geq\left(1-32 \delta_{0}^{2}-64 \delta_{0}^{2} \frac{\Lambda_{0}}{(n-2) \lambda_{0}}\right) \frac{|\nabla u|^{4}}{4} \sum_{i=2}^{n-1} F^{i i} \\
& \geq\left(1-32 \delta_{0}^{2}-64 \delta_{0}^{2} \frac{\Lambda_{0}}{(n-2) \lambda_{0}}\right) \frac{(n-2) \lambda_{0}}{n \Lambda_{0}} \frac{|\nabla u|^{4}}{4} \sum_{i=2}^{n-1} F^{i i},
\end{aligned}
$$

Case 2. There is no $j \in I$ satisfying (30).

For this case, the proof is the same as in [16. We repeat it here for completeness.

We may assume that there is $i_{0}$ such that $\tilde{u}_{i_{0} i_{0}}^{2} \leq \delta_{0}^{2}|\nabla u|^{4}$, otherwise the claim is automatically true. Assume $i_{0}=1$. As in Case 4 in [15], we have $u_{1}^{2} \geq\left(1-2 \delta_{0}\right)|\nabla u|^{2}$ and $\tilde{u}_{j j}^{2}+u_{j}^{2}\left(|\nabla u|^{2}-u_{j}^{2}\right) \geq$ $\delta_{0}^{2}|\nabla u|^{4}$ for $j>1$. From equation (29), we have

$$
\begin{aligned}
\sum_{i, l} F^{i i} \tilde{u}_{i l}^{2} & =\sum_{i} F^{i i}\left\{\tilde{u}_{i i}^{2}+u_{i}^{2}\left(|\nabla u|^{2}-u_{i}^{2}\right)\right\} \\
& \geq \sum_{i \geq 2} F^{i i}\left(\tilde{u}_{i i}^{2}+u_{i}^{2}\left(|\nabla u|^{2}-u_{i}^{2}\right)\right) \\
& \geq \delta_{0}^{2}|\nabla u|^{4} \sum_{i \geq 2} F^{i i} \geq C \delta_{0}^{2}|\nabla u|^{4} \sum_{i \geq 1} F^{i i} .
\end{aligned}
$$

The latter inequality follows from the uniformly ellipticity of $F$. This finishes the proof the Claim and hence the Proposition.

We have a direct corollary.

Corollary 3. Suppose $F$ is a $C^{2}$ concave and uniformly elliptic operator with ellipticity constants $\lambda_{0}, \Lambda_{0}$. Let $B_{1}$ be a unit disk in a compact Riemannian manifold $M$ and $u$ a $C^{2}$ solution of equation

$$
F\left(\nabla^{2} u+d u \otimes d u-\frac{|\nabla u|^{2}}{2} g_{0}+S_{g_{0}}\right)=e^{-2 u} f(x), \quad x \in B_{1}
$$

for a $C^{1}$ function $f: B_{1} \rightarrow \mathbb{R}$. Then there is a constant $C>0$ depending only on $\lambda_{0}, \Lambda_{0}, g_{0}$ such that

$$
|\nabla u|^{2}(x) \leq C\left(1+\|f\|_{C^{1}\left(B_{1}\right)} e^{-2 \inf _{B_{1}} u}\right), \quad \text { for any } x \in B_{1 / 2} .
$$

Proof. We pick $\rho \in C_{0}^{2}\left(B_{1}\right)$ such that $\rho(x)=1, \forall x \in B_{\frac{1}{2}}$ and $0 \leq \rho(x) \leq 1, \forall x \in B_{1}$. (34) follows directly from (20) with $\tilde{f}=e^{-2 u} f$. 
In what follows in the next sections, we will only need Corollary 3 as we will work on smooth operator $F$. We note that estimates (34) and (18) are independent of the smoothness of $F$. Theorem 5 can also be proved by certain appropriate approximations.

$A$ sketch proof of Theorem 5. Since $u \in C^{2}, u$ is in fact $C^{2, \alpha}$ by the Evans-Krylov theorem. We may find two sequences of smooth functions $\left\{u_{k}\right\}$ and $\left\{f_{k}\right\}$, such that $u_{k} \rightarrow u$ in $C^{2, \alpha}\left(\bar{B}_{1}\right)$, $f_{k} \rightarrow f$ in $C^{0,1}\left(\bar{B}_{1}\right)$, and

$$
F\left(\nabla^{2} u_{k}+d u_{k} \otimes d u_{k}-\frac{\left|\nabla u_{k}\right|^{2}}{2} g_{0}+S_{g_{0}}\right) \geq e^{-2 u_{k}} f_{k} .
$$

We now construct a sequence of smooth concave $F_{k}: \mathcal{S} \rightarrow \mathbb{R}$ such that $F_{k}$ converges to $F$ uniformly in compacts of $\mathcal{S}$ and $F_{k}$ is uniformly elliptic with ellipticity constants $\frac{\lambda_{0}}{2}$ and $2 \Lambda_{0}$. We may assume

$$
F_{k}(\lambda) \geq F(\lambda), \quad \forall|\lambda| \leq \sup _{B_{1}}\left|\nabla^{2} u+d u \otimes d u-\frac{|\nabla u|^{2}}{2} g_{0}+S_{g_{0}}\right|+1 .
$$

By the symmetry of $F_{k}, \frac{\partial F_{k}(1, \cdots, 1)}{\partial \lambda_{i}}=\frac{\partial F_{k}(1, \cdots, 1)}{\partial \lambda_{j}}$ for all $i, j$. Set $\frac{\partial F_{k}(1, \cdots, 1)}{\partial \lambda_{j}}=A$. Let $R_{0}(x)$ be the scalar curvature of $g_{0}$, and let $\tilde{u}$ be the solution of $\Delta \tilde{u}_{k}=\frac{f_{k} e^{-2 u_{k}}}{A}-R_{0}+n-\frac{F_{k}(1, \cdots, 1)}{A}$ in $B_{1}$ with $\tilde{u}_{k}=u_{k}$ on $\partial B_{1}$. We consider the following Dirichlet problem

$$
\begin{aligned}
F_{k}\left(\nabla^{2} v_{k}+d v_{k} \otimes d v_{k}-\frac{\left|\nabla v_{k}\right|^{2}}{2} g_{0}+S_{g_{0}}\right) & =f_{k} e^{-2 u_{k},}, & & \text { in } B_{1}, \\
v_{k} & =u_{k}, & & \text { on } \partial B_{1} .
\end{aligned}
$$

By the concavity of $F_{k}$,

$$
F_{k}(\lambda) \leq A \sigma_{1}(\lambda)-n A+F_{k}(1, \cdots, 1)
$$

We have

$$
\begin{aligned}
A\left(\Delta v_{k}+R_{0}-n+\frac{F_{k}(1, \cdots, 1)}{A}\right) & \geq A\left(\Delta v_{k}-\frac{n-2}{2}\left|\nabla v_{k}\right|^{2}+R_{0}-n+\frac{F_{k}(1, \cdots, 1)}{A}\right) \\
& \geq f_{k} e^{-2 u_{k}} \\
& \geq F_{k}\left(\nabla^{2} v_{k}+d v_{k} \otimes d v_{k}-\frac{\left|\nabla v_{k}\right|^{2}}{2} g_{0}+S_{g_{0}}\right) .
\end{aligned}
$$

In turn, we have $v_{k} \leq \tilde{u}_{k}$ in $B_{1}$. On the other hand, we have

$$
\begin{aligned}
F_{k}\left(\nabla^{2} u_{k}+d u_{k} \otimes d u_{k}-\frac{\left|\nabla u_{k}\right|^{2}}{2} g_{0}+S_{g_{0}}\right) & \geq F\left(\nabla^{2} u_{k}+d u_{k} \otimes d u_{k}-\frac{\left|\nabla u_{k}\right|^{2}}{2} g_{0}+S_{g_{0}}\right) \\
& =F_{k}\left(\nabla^{2} v_{k}+d v_{k} \otimes d v_{k}-\frac{\left|\nabla v_{k}\right|^{2}}{2} g_{0}+S_{g_{0}}\right) .
\end{aligned}
$$

This gives $v_{k} \geq u_{k}$ in $B_{1}$. From this, we obtain a $C^{0}$ bound of $v_{k}$ and a bound of $\left|\nabla v_{k}\right|$ at the boundary $\partial B_{1}$. Using the same proof of Proposition 11, we can obtain a bound of $\left|\nabla v_{k}\right|$ on $\bar{B}_{1}$ (simply let $H(x)=\left|\nabla v_{k}\right|^{2}$ and estimate at the maximum point if it is not on the boundary). At this end, we have a uniform $C^{1}$ bound of $v_{k}$. The standard barrier construction $\omega_{ \pm}$similar to the one in Step 3 in Chapter 9 of [] (page 91), with the modified operator $\tilde{F}\left(\omega_{ \pm}\right)=F_{k}\left(\nabla^{2} \omega_{ \pm}+\right.$ 
$d v_{k} \otimes d v_{k}-\frac{\left|\nabla v_{k}\right|^{2}}{2} g_{0}+S_{g_{0}}$ ), will give a $C^{2}$ bound near boundary. The global $C^{2}$ estimate follows easily along the lines of proof in Proposition 3.1 in [15] (see also proof of Lemma 3 in [14]). Higher regularity estimates follow from the Krylov Theorem [21]. We note that $C^{2}$ and higher regularity bounds of $v_{k}$ may depend on higher smoothness assumptions on $u_{k}$ and $f_{k}$, but the interior $C^{2, \alpha}$ estimates of $v_{k}$ depend only on $\left\|u_{k}\right\|_{C^{2, \alpha}\left(\bar{B}_{1}\right)}$ and $\left\|f_{k}\right\|_{C^{1}\left(\bar{B}_{1}\right)}$ by Theorem 4 . In any case, we can establish the existence of the Dirichlet problem (35) by using the method of continuity for equation

$$
F_{t}\left(\nabla^{2} v_{k}+d v_{k} \otimes d v_{k}-\frac{\left|\nabla v_{k}\right|^{2}}{2} g_{0}+S_{g_{0}}\right)=t f_{k} e^{-2 u_{k}}+(1-t) f_{k}^{*},
$$

where $F_{t}(\lambda)=t F_{k}(\lambda)+(1-t) \sigma_{1}(\lambda)$ and $f_{k}^{*}=\sigma_{1}\left(\nabla^{2} u_{k}+d u_{k} \otimes d u_{k}-\frac{\left|\nabla u_{k}\right|^{2}}{2} g_{0}+S_{g_{0}}\right)$. Now by Proposition 11 we have for any $\rho \in C_{0}^{2}\left(B_{1}\right)$ with $0 \leq \rho \leq 1$, there exists $C$ independent of $k$ and $u_{k}$ such that

$$
\max _{B_{1}}\left\{\left|\rho(x) \nabla v_{k}\right|^{2}(x)\right\} \leq C\left(1+\left(\max _{B_{1}}\left\{\left|\rho(x) f_{k}(x)\right|\left|\nabla u_{k}(x)\right|\right\}+\max _{B_{1}}\left\{\rho(x)\left|\nabla f_{k}(x)\right|\right\}\right) e^{-2 u_{k}(x)}\right) .
$$

From our estimates, $\left\|v_{k}\right\|_{C^{1}\left(\bar{B}_{1}\right)} \leq C$, where $C$ is a constant independent of $k$, since $\left\|u_{k}\right\|_{C^{2, \alpha}\left(\bar{B}_{1}\right)}$ and $\left\|f_{k}\right\|_{C^{1}\left(\bar{B}_{1}\right)}$ are uniformly bounded. By Theorem $4 v_{k} \rightarrow v_{0}$ (after passing a subsequence) in $C^{2, \alpha}\left(B_{1}\right), v_{k}$ converges to $v_{0}=u$ by the uniqueness. Therefore, there is a constant $C>0$ depending only on $\lambda_{0}, \Lambda_{0}$, and the geometry of $B_{1}$ such that

$$
\max _{B_{\frac{1}{2}}}|\nabla u|^{2}(x) \leq C\left(1+\|f\|_{C^{1}\left(B_{1}\right)} e^{-2 \inf _{B_{1}} u(x)}\right) .
$$

This finishes the proof of Theorem 5 .

A direct consequence of Theorem [5 is the following

Corollary 4. Let $B_{1}$ be a unit disk in a Riemannian manifold $\left(M, g_{0}\right)$ and $p<n / 2$. There exists a small constant $\varepsilon_{0}>0$ depending only on $\left(B_{1}, g_{0}\right)$ such that for any sequence of solutions $u_{i}$ of (2) in $B_{1}$ with

$$
\int_{B_{1}} e^{-n u_{i}} d v o l\left(g_{0}\right) \leq \varepsilon_{0}
$$

either

(1) there is a subsequence $u_{i_{l}}$ uniformly converging to $+\infty$ in any compact subset of $B_{1}$, or

(2) there is a subsequence $u_{i_{l}}$ converging strongly in $C_{l o c}^{2, \alpha}\left(B_{1}\right)$.

Proof. It follows the same lines of proof in [36] or [15].

\section{NEGATIVE CURVATURE CASE}

In this section, we discuss the negative curvature case, where the geometry is rich. By [1] $(n=3)$ and 27] (general dimension $n \geq 3$ ), every higher dimensional manifold has a metric with negative Ricci tensor. It is clear that such a metric also has negative $W_{p}$ for any $1 \leq p \leq n-1$. Hence every higher dimensional manifold has a metric with negative $W_{p}$. The 
conformal deformation will yield interesting geometric information about the extremal metrics in a given conformal class.

Proof of Theorem 1. First we take the sequence of smooth $F_{k}$ considered in the previous section. For each $F_{k}$ we consider the following equation

$$
F_{k}\left(\left(e^{2 u}\left(\nabla^{2} u+d u \otimes d u-\frac{|\nabla u|^{2}}{2} g_{0}+S_{g_{0}}\right)\right)=-1 .\right.
$$

For large $k$, from the condition of the Theorem, we have

$$
F_{k}\left(S_{g_{0}}\right)(x)<0, \quad \forall x \in M .
$$

We first prove the existence of solutions to equation (37). Here we use the method of continuity. Let us consider the following equation

$$
F_{t}(u):=F_{k}\left(e^{2 u}\left(\nabla^{2} u+d u \otimes d u-\frac{|\nabla u|^{2}}{2} g_{0}+S_{g_{0}}\right)\right)+t-(1-t) F_{k}\left(S_{g_{0}}\right)=0
$$

and define $J=\{t \rightarrow[0,1] \mid(38)$ has a solution for $t\}$. It is clear that $0 \in J$. First, we prove the openness of $J$. Let $t_{0} \in J$. By the maximum principle, we know that there is only one solution $u$ of (38) for $t=t_{0}$. Let $L$ be its linearization. We want to show that $L$ is invertible. By the maximum principle again, we know that the kernel of $L$ is trivial. Note that $L$ might be not self-adjoint. To show the invertibility of $L$, we need to show that the cokernel of $L$ is also trivial. However, one can readily check that the Fredholm index of $L$ is zero, and hence the cokernel of $L$ is trivial. Now the openness follows from the implicit function theorem.

Then we show the closeness. Let $x_{0}$ and $x_{1}$ be the minimum and maximum of $u$ respectively. By the maximum principle, we have

$$
e^{2 u\left(x_{0}\right)} \geq \frac{t-(1-t) F_{k}\left(S_{g_{0}}\right)\left(x_{0}\right)}{-F_{k}\left(S_{g_{0}}\right)\left(x_{0}\right)}
$$

and

$$
e^{2 u\left(x_{1}\right)} \leq \frac{t-(1-t) F_{k}\left(S_{g_{0}}\right)\left(x_{1}\right)}{-F_{k}\left(S_{g_{0}}\right)\left(x_{1}\right)}
$$

Hence, we have $C^{0}$ bound of $u$ independent of $t$.

By a global estimates proven in Proposition 2 below and Theorem 4, we have the closeness. Hence we have a solution $u_{k}$ of (37) with the bound

$$
\frac{1}{-\min F_{k}\left(S_{g_{0}}\right)\left(x_{0}\right)} \leq e^{2 u_{k}} \leq \frac{1}{-\max F_{k}\left(S_{g_{0}}\right)\left(x_{0}\right)} .
$$

In viewing of (41), we use again the global estimates and Theorem 4 to obtain a $C^{2, \alpha}$ uniform bound of $u_{k}$ for some $\alpha>0$. Hence $u_{k}$ converges (by taking a subsequence) to $u$. It is clear that $e^{-2 u} g_{0}$ satisfies (2).

Proposition 2. Let $u$ be a solution of

$$
F\left(\nabla^{2} u+d u \otimes d u-\frac{|\nabla u|^{2}}{2} g_{0}+S_{g_{0}}\right)=f e^{-2 u}
$$


with $C^{1}$ function $f$. Suppose that $F$ is uniformly elliptic and is homogeneous of degree 1. Assume that $u$ has $C^{0}$ bound. Then $u$ has a $C^{1}$ bound and a $C^{2, \alpha}$ bound.

Proof. Since we already have full $C^{0}$ bound, the proposition can be proved using standard Pogorelov type of trick, for example, as in [19]. Let $v=e^{u}$ and consider the following equivalent form of (42)

$$
F\left(\frac{\nabla^{2} v}{v}-\frac{|\nabla v|^{2}}{v^{2}} g_{0}+S_{g_{0}}\right)=f v^{-2}
$$

Without loss of generality, we may assume that $v \leq 1$. Set $H=e^{2 \phi(v)}|\nabla v|^{2}$. Here $\phi$ will be fixed later. Let $x_{0}$ be a maximum point of $H$. At $x_{0}$, we have

$$
\sum_{k}\left(2 v_{k} v_{k i}+2 \phi^{\prime}(v) v_{i}|\nabla v|^{2}\right)=0, \quad \text { for any } i \text {. }
$$

Without loss of generality, we may assume that $v_{1}=|\nabla v|, v_{i}=0$ for any other $i$ and that $v_{i j}$ is diagonal at $x_{0}$. Hence, (44) is equivalent to $v_{11}=-\phi^{\prime}(v)|\nabla v|^{2}$. Set $w_{i j}=v_{i j}-\frac{|\nabla v|^{2}}{2 v} \delta_{i j}+v S_{i j}$. The maximum principle, together with (44), implies

$$
0 \geq \sum F^{i j}\left(v_{k j} v_{k i}+v_{k} v_{k i j}+\phi^{\prime}(v) v_{i j}|\nabla v|^{2}+v_{i} v_{j} \phi^{\prime \prime}(v)|\nabla v|^{2}+2 \phi^{\prime}(v) v_{i} v_{k i} v_{k}\right)
$$

By (44), it is easy to check that

$$
\begin{aligned}
& \sum F^{i j} v_{k i} v_{k j} \geq F^{11} v_{11} v_{11}=F^{11}\left(\phi^{\prime}(v)\right)^{2} \frac{|\nabla v|^{4}}{v} \\
& \sum F^{i j} v_{k} v_{k i j}= \sum F^{i j} v_{k} v_{i j k}+\sum F^{i j} v_{k} v_{m} R_{i j k}^{m} \\
& \geq \sum F^{i j} v_{k}\left(w_{i j}+\frac{|\nabla v|^{2}}{2 v} \delta_{i j}+v S_{i j}\right)_{k}-C \max v|\nabla v|^{2} \\
& \geq 2 v_{k}\left(f v^{-1}\right)_{k}+\sum F^{i i} v_{1}\left(-\frac{v_{1}^{3}}{2 v^{2}}-\phi^{\prime}(v) \frac{|\nabla v|^{3}}{v}\right)-C \max v|\nabla v|^{2} \\
& \geq 2 f_{k} v_{k}-2 v^{-1}|\nabla v|^{2}-\left(\frac{1}{2 v}+\phi^{\prime}(v)\right) \sum F^{i i} \frac{|\nabla v|^{4}}{v}-C \max v|\nabla v|^{2}, \\
& \phi^{\prime}(v) \sum F^{i j} v_{i j}|\nabla v|^{2}=\phi^{\prime}(v) \sum F^{i j}\left(w_{i j}+\frac{|\nabla v|^{2}}{2 v} \delta_{i j}-v S_{i j}\right)|\nabla v|^{2} \\
& \geq \phi^{\prime}(v) f v^{-1}|\nabla v|^{2}+\phi^{\prime}(v) \sum F^{i i} \frac{|\nabla v|^{4}}{2 v}-C \max v|\nabla v|^{2}, \\
& \phi^{\prime \prime}(v) \sum F^{i j} v_{i} v_{j}|\nabla v|^{2}=\phi^{\prime \prime}(v) F^{11}|\nabla v|^{4} \\
& 2 \phi^{\prime}(v) \sum F^{i j} v_{j} v_{k} v_{k i}=2 \phi^{\prime}(v) F^{11} v_{1}^{2} v_{11}=-2\left(\phi^{\prime}(v)\right)^{2} F^{11}|\nabla v|^{4} .
\end{aligned}
$$


Here $C$ is a positive constant depending only on the Riemannian curvature of the background metric and it varies from line to line. Therefore, we have

$$
\begin{aligned}
0 \geq & F^{11}\left(\phi^{\prime \prime}(v)-\left(\phi^{\prime}(v)\right)^{2}\right)|\nabla v|^{4}+\frac{1}{2 v} \sum F^{i i}\left(-\frac{1}{v}+\phi(v)\right)|\nabla v|^{4}-C \max v|\nabla v|^{2} \\
& +2 f_{k} v_{k}-2 v^{-1}|\nabla v|^{2}+\phi^{\prime}(v) f v^{-1}|\nabla v|^{2}
\end{aligned}
$$

Choose

$$
\phi=-\frac{1}{2} \log t(c t-2),
$$

for a large constant $c>0$ so that $c \min v>3$. One can easily check that for any $t \in[\min v, \infty)$

$$
\phi^{\prime \prime}(v)-\left(\phi^{\prime}(v)\right)^{2}=\frac{1}{t^{2}(c t-2)^{2}}>0
$$

and

$$
-\left(\frac{1}{t}+\phi(t)\right)=\frac{1}{t(c t-2)}
$$

In view of (46), we have

$$
0 \geq \frac{1}{2(\max v)^{2}(c \max v-2)}|\nabla v|^{4} F^{i i}-c(f, C)|\nabla v|^{2} .
$$

Now we have a global bound of $|\nabla v|$, which depend only on $g_{0}, f, \min v$ and $\max v$. The $C^{2, \alpha}$ bound follows from Theorem 4 .

Remark 1. The condition $W_{p}\left(g_{0}\right)(x)<0, \forall x \in M$ in Theorem 1 can be weaken to $W_{p}\left(g_{0}\right)(x) \leq$ $0, \forall x \in M$ and $W_{p}\left(g_{0}\right)\left(x_{0}\right)<0$ for some $x_{0} \in M$. In fact, under the weaker condition, one may produce a metric $g \in\left[g_{0}\right]$ with the stronger condition holds. This can be done using the short time existence of the fully nonlinear flow

$$
u_{t}=G_{p}\left(\nabla^{2} u+d u \otimes d u-\frac{|\nabla u|^{2}}{2} g_{0}+S_{g_{0}}\right) e^{2 u},\left.\quad u\right|_{t=0}=0 .
$$

The short time existence follows from standard nonlinear parabolic theory, and the strict negativity of $W_{p}(g)$ (which is equal to $u_{t}$ ) follows from the strong maximum principle.

Remark 2. It is of interest to characterize when the condition in Theorem 1 is true by some conformal geometric quantities. The difficulty here is the lack of variational structure for this type of equations. We note that when $p>\frac{n}{2}$, if the Yamabe constant $Y\left(\left[g_{0}\right]\right)$ of $\left(M, g_{0}\right)$ is nonpositive, then the condition in Theorem 1 is satisfied unless $\left(M, g_{0}\right)$ is conformally equivalent to a Ricci flat manifold. This simple observation follows from the solution of the Yamabe problem and the fact that if the scalar curvature vanishing identically, for $p>\frac{n}{2}, G_{p} \leq 0$ and $G_{p}$ vanishes identically if and only if the metric is Ricci flat.

Remark 3. It is also an interesting problem to consider the equation $W_{p}(g)=-1$ on a complete, non-compact manifold. The arguments in the proof of the existence of Dirichlet problem (35) can be extended to deal with a given boundary condition at the infinity for equation $W_{p}(g)=-1$ on a complete non-compact negatively curved manifold. 
As a direct consequence of Theorem 1 we have

Corollary 5. If there is $\tilde{g} \in\left[g_{0}\right]$ with $\mathcal{R}_{\tilde{g}}^{s}(x)<0$ for all $x \in M$ then there is a unique $C^{2, \alpha}$ metric $g^{*} \in\left[g_{0}\right]$ for some $\alpha>0$ such that $\mathcal{R}_{g^{*}}^{s}(x)=-1, \forall x \in M$. Similarly, If there is $\tilde{g} \in\left[g_{0}\right]$ with $\mathcal{R}_{\tilde{g}}^{l}(x)<0$ for all $x \in M$, then there is a unique $g_{*} \in\left[g_{0}\right]$ and $g_{*} \in C^{2, \alpha}(M)$ for some $\alpha>0$ such that $\mathcal{R}_{g_{*}}^{l}(x)=-1, \forall x \in M$.

Corollary [5 can be applied to consider minimal volumes in conformal classes. Set

$$
\begin{aligned}
& \mathcal{C}^{-}=\left\{g \in\left[g_{0}\right] \mid \mathcal{R}_{g}^{s}(x) \geq-1, \forall x \in M\right\}, \\
& \mathcal{C}_{-}=\left\{g \in\left[g_{0}\right] \mid \mathcal{R}_{g}^{l}(x) \geq-1, \forall x \in M\right\}, \\
& \tilde{\mathcal{C}}^{-}=\left\{g \in\left[g_{0}\right] \mid \mathcal{R}_{g}^{s}(x) \leq-1, \forall x \in M\right\}, \\
& \tilde{\mathcal{C}}_{-}=\left\{g \in\left[g_{0}\right] \mid \mathcal{R}_{g}^{l}(x) \leq-1, \forall x \in M\right\} .
\end{aligned}
$$

Define

$$
\begin{array}{rlrl}
\mathcal{V}^{s}\left(\left[g_{0}\right]\right) & =\inf _{g \in \mathcal{C}^{-}} \operatorname{vol}(g), & \mathcal{V}^{l}\left(\left[g_{0}\right]\right)=\inf _{g \in \mathcal{C}_{-}} \operatorname{vol}(g), \\
\mathbb{V}^{s}\left(\left[g_{0}\right]\right)=\sup _{g \in \tilde{\mathcal{C}}^{-}} \operatorname{vol}(g), & \mathbb{V}^{l}\left(\left[g_{0}\right]\right)=\sup _{g \in \tilde{\mathcal{C}}_{-}} \operatorname{vol}(g),
\end{array}
$$

where $\operatorname{vol}(g)$ is the volume of $g$. It is clear that the definitions given here

Lemma 1. Let $g_{1}, g, g_{2} \in\left[g_{0}\right]$. If $\mathcal{R}_{g}^{s}(x)<0$, and $\mathcal{R}_{g_{1}}^{s}(x) \leq \mathcal{R}_{g}^{s}(x) \leq \mathcal{R}_{g}^{s}(x), \forall x \in M$, then $\operatorname{vol}\left(g_{1}\right) \leq \operatorname{vol}(g) \leq \operatorname{vol}\left(g_{2}\right)$, any one of the equalities holds if and only if the metric is equal to $g$. Similarly, if $\mathcal{R}_{g}^{l}(x)<0$, and $\mathcal{R}_{g_{1}}^{l}(x) \leq \mathcal{R}_{g}^{l}(x) \leq \mathcal{R}_{g}^{l}(x), \forall x \in M$, then $\operatorname{vol}\left(g_{1}\right) \leq \operatorname{vol}(g) \leq \operatorname{vol}\left(g_{2}\right)$, any one of the equalities holds if and only if the metric is equal to $\mathrm{g}$.

The Lemma is a simple consequence of the maximum principle applied to equations (7) or (8). From the lemma, we have the following relations

$$
\mathcal{V}^{s}\left(\left[g_{0}\right]\right) \geq \mathbb{V}^{s}\left(\left[g_{0}\right]\right) \geq \mathcal{V}^{l}\left(\left[g_{0}\right]\right) \geq \mathbb{V}^{l}\left(\left[g_{0}\right]\right)
$$

And we can show that the minimal volumes $\mathcal{V}^{s}\left(\left[g_{0}\right]\right)$ and $\mathcal{V}^{l}\left(\left[g_{0}\right]\right)$ are achieved.

Corollary 6. Suppose that $\mathcal{R}_{g_{0}}^{s}(x)<0$ for any $x \in M$. Then there is a unique conformal metric $g^{*} \in\left[g_{0}\right]$ such that $\operatorname{vol}\left(g^{*}\right)=\mathcal{V}^{s}\left(\left[g_{0}\right]\right)$ with $\mathcal{R}_{g^{*}}^{s}(x)=-1, \forall x \in M$ and

$$
\mathbb{V}^{s}\left(\left[g_{0}\right]\right)=\mathcal{V}^{s}\left(\left[g_{0}\right]\right) \geq \mathcal{V}^{l}\left(\left[g_{0}\right]\right) .
$$

The equality holds if and only if there is an Einstein metric in $\left[g_{0}\right]$. If $\mathcal{R}_{g_{0}}^{l}(x)<0$ for any $x \in M$, then there is a unique $g^{*} \in\left[g_{0}\right]$ such that $\operatorname{vol}\left(g_{*}\right)=\mathcal{V}^{l}\left(\left[g_{0}\right]\right)$ with $\mathcal{R}_{g_{*}}^{l}(x)=-1, \forall x \in M$. In this case, we have

$$
\mathcal{V}^{s}\left(\left[g_{0}\right]\right)=\mathbb{V}^{s}\left(\left[g_{0}\right]\right) \geq \mathcal{V}^{l}\left(\left[g_{0}\right]\right)=\mathbb{V}^{l}\left(\left[g_{0}\right]\right) .
$$

For the study of minimal volumes in general Riemannian manifolds, we refer to [12, 4] and [23]. 


\section{POSITIVE CURVATURE CASE}

Now we consider conformal classes with metrics of positive Ricci curvature. Let $\left[g_{0}\right]$ be such a conformal class. After a suitable scaling, we may assume that $R i c_{g_{0}} \geq(n-1) g_{0}$. Define $\left[g_{0}\right]_{+}=\left\{g \in\left[g_{0}\right] \mid R i c_{g} \geq(n-1) g\right\}$ and $V_{\max }\left(M,\left[g_{0}\right]\right)=\sup _{g \in\left[g_{0}\right]_{+}} \operatorname{vol}_{g}(M)$. This definition is motivated by Gursky and Viaclovsky [18. From the Bishop comparison, we know

$$
V_{\max }\left(M,\left[g_{0}\right]\right) \leq \operatorname{vol}\left(\mathbb{S}^{n}\right)
$$

the volume of the unit sphere.

Proposition 3. Let $\left(M, g_{0}\right)$ be a compact Riemannian manifold with Ric $g_{g_{0}} \geq(n-1) g_{0}$. If $V_{\max }\left(M,\left[g_{0}\right]\right)<\operatorname{vol}\left(\mathbb{S}^{n}\right)$, then there is a conformal metric $g \in\left[g_{0}\right]_{+}$with

$$
\mathcal{R}_{g}^{s}=n-1 .
$$

Proof. Consider the sequence of approximating function $F_{k}$ as in Section 2 with a normalization condition that $\left.F_{k}(1,1, \cdot, 1)=n-1\right)$. We first want to find a solution to the following equation

$$
F_{k}\left(e^{2 u}\left(\nabla^{2} u+d u \otimes d u-\frac{|\nabla u|^{2}}{2} g_{0}+S_{g_{0}}\right)\right)=n-1,
$$

for large $k$. Define

$$
V_{\max }^{k}\left(\left[g_{0}\right]\right)=\max \left\{\operatorname{vol}(g) \mid g \in\left[g_{0}\right] \text { with } F_{k}\left(g^{-1} \cdot S_{g}\right) \geq n-1\right\} .
$$

It is easy to check that $\lim _{k \rightarrow \infty} V_{\max }^{k}\left(\left[g_{0}\right]\right)=V_{\max }\left(\left[g_{0}\right]\right)$. Hence for large $k$ we have

$$
V_{\max }^{k}\left(\left[g_{0}\right]\right)<\operatorname{vol}\left(\mathbb{S}^{n}\right) .
$$

To show the existence of solution of (48), we consider a deformation, which is similar to a deformation considered by Gursky and Viaclovsky in their study of $\sigma_{k}$-Yamabe problem.

$$
\begin{array}{r}
F_{k}\left(\nabla^{2} u+d u \otimes d u-\frac{1}{2}|\nabla u|^{2} g+\psi(t) S_{g}+(1-\psi) g\right) \\
=(n-1)(1-t)\left(\frac{1}{\operatorname{vol}\left(g_{0}\right)} \int_{M} e^{-(n+1) u}\right)^{\frac{2}{n+1}}+(n-1) \psi(t) e^{-2 u},
\end{array}
$$

where $\psi(t):[0,1] \rightarrow[0,1]$ is a $C^{1}$ function satisfying $\psi(0)=0$ and $\psi(t)=1$ for $t \geq 1 / 2$. We now prove that there is a solution of (50) when $t=1$, provided that $V_{\max }^{k}(M,[g])<\operatorname{vol}\left(\mathbb{S}^{n}\right)$.

When $t=0$, it is easy to check that (50) has a unique solution $u=0$ and its corresponding linearization has no nontrivial kernel. Hence its Leray-Schauder degree is non-zero. If the solution space of (50) for any $t \in[0,1]$ is compact, then using degree theoretic argument, we are done. Assume by contradiction that there is no compactness. Assume without loss of generality that there is a sequence of solutions $g_{i}=e^{-2 u_{i}} g$ of (1) with $t=1$ such that $u_{i}$ does not converge in $C^{2, \alpha}$. In view of Theorem 5 and Corollary 2$]$ we have either
(a) $\inf _{M} u_{i} \rightarrow-\infty$, or
(b) $\inf _{M} u_{i} \rightarrow+\infty$. 
The latter is easy to be excluded as follows. At the minimum point of $u_{i}$, we have

$$
e^{2 \inf u_{i}} F_{k}\left(S_{g_{0}}\right)=F_{k}\left(e^{2 \inf u_{i}} S_{0}\right) \leq(n-1),
$$

which certainly implies that inf $u_{i}$ is bounded from above uniformly. Hence we are left to exclude (a). Let $x_{i}$ be the minimum point of $u_{i}$ and assume that $x_{i} \rightarrow x_{0}$ as $i \rightarrow \infty$. Consider a scaled function

$$
\tilde{u}_{i}=u\left(\exp _{x_{i}} \varepsilon_{i} x\right)-\log \varepsilon_{i},
$$

where $\varepsilon_{i}=\exp u_{i}\left(x_{i}\right)$. It is clear that $\tilde{u}_{i} \geq 0$ satisfies a similar equation on $B\left(0, \varepsilon_{i}^{-1} r_{0} / 2\right) \subset \mathbb{R}^{n}$ with a scaled metric, where $r_{0}$ is the injectivity radius of $(M, g)$. The set $B\left(0, \varepsilon_{i}^{-1} r_{0} / 2\right)$ with the scaled metric converges to $\mathbb{R}^{n}$. By local estimates and local $C^{2}$ estimates in 14 for a more general concave case one can show that $\tilde{u}_{i}$ converges to an entire solution $v$ of

$$
F_{k}\left(\nabla^{2} v+d v \otimes d v-\frac{1}{2}|\nabla v|^{2} g_{\mathbb{R}^{n}}\right)=(n-1) e^{-2 v}
$$

and

$$
V_{\max }^{k}(g) \geq \liminf _{i} \operatorname{vol}\left(e^{-2 u_{i}} g\right) \geq \operatorname{vol}\left(\mathbb{R}^{n}, e^{-2 v} g_{\mathbb{R}^{n}}\right),
$$

where $g_{\mathbb{R}^{n}}$ is the standard Euclidean metric. By a classification result of Li-Li in 26], we know that $\left(\mathbb{R}^{n}, e^{-2 v} g_{\mathbb{R}^{n}}\right)$ is equivalent to $\mathbb{S}^{n}$. Hence $V_{\max }^{k}\left(\left[g_{0}\right]\right) \geq \operatorname{vol}\left(\mathbb{R}^{n}, e^{-2 v} g_{\mathbb{R}^{n}}\right)=\operatorname{vol}\left(\mathbb{S}^{n}\right)$. This contradicts (49). Therefore, we have a solution $u_{k}$ of (50) for large $k$.

Now we consider the sequence $\left\{u_{k}\right\}$. As above, we can show first that $u_{k}$ has a uniform upper bound. If $u_{k}$ has a uniform lower bound, Corollary 2 implies that the sequence $\left\{u_{k}\right\}$ has a uniform $C^{2, \alpha}$ bound. And hence we have a limit $u_{0}$ which is a solution we desire. Hence to prove the Proposition, we only need to exclude the case that $\min u_{k} \rightarrow-\infty$. Assume that we are in this case. By a similar argument presented above, after considering a suitable rescaling we have a limit $C^{2, \alpha}$ function $v_{\infty}$ satisfying

$$
G_{n-1}\left(\nabla^{2} v+d v \otimes d v-\frac{1}{2}|\nabla v|^{2} g_{\mathbb{R}^{n}}\right)=(n-1) e^{-2 v}
$$

and

$$
V_{\max }^{k}\left(\left[g_{0}\right]\right) \geq \liminf _{i} \operatorname{vol}\left(e^{-2 u_{i}} g\right) \geq \operatorname{vol}\left(\mathbb{R}^{n}, e^{-2 v_{\infty}} g_{\mathbb{R}^{n}}\right) .
$$

The contradiction follows from the following Lemma. We finish the proof of the Proposition.

Proposition 4. Let $p<n / 2$ and $g=e^{-2 u} g_{\mathbb{R}^{n}}$ be a $C^{2}$ function on $\mathbb{R}^{n}$ such that

$$
G_{p}\left(g^{-1} S_{g}\right)(x)=c, \forall x \in \mathbb{R}^{n},
$$

for some constant $c$. Then $u=0$ if $c \leq 0$ and $u(x)=\log \frac{\lambda^{2}+\left|x-x_{0}\right|^{2}}{2 \lambda \sqrt{\frac{(n-p) p}{c}}}$ if $c>0$. That is, $\left(\mathbb{R}^{n}, e^{-2 u} g_{\mathbb{R}^{n}}\right)$ can be compactified as a standard sphere if $c>0$.

Proof. The proof follows 26] closely. The only difference is that the operator there is required to be $C^{1}$. Here our operator $G_{p}$ is Lipschitz only. However $G_{p}$ is uniformly elliptic and concave. We will show in Lemma 2 below that the Hopf lemma holds for our equation. Then the argument in [26] can be applied to our equation. 
Lemma 2. Let $\Omega$ be a bounded domain in $\mathbb{R}^{n}$ and $p<n / 2$. If (9) has two solutions $w$ and $v$ with $w \geq v$ and $w\left(x_{0}\right)=v\left(x_{0}\right)$ for some $x_{0} \in \partial \Omega$, then $x_{0} \in \partial \Omega$. Furthermore,

$$
\frac{\partial w}{\partial \nu}\left(x_{0}\right)<\frac{\partial v}{\partial \nu}\left(x_{0}\right)
$$

unless $w=v$. Here $\nu$ is the outer normal of $\partial \Omega$ at $x_{0}$.

Proof. For any function, set

$$
A^{u}=\nabla u+d u \otimes d s-\frac{1}{2}|\nabla u|^{2} g_{0}+S_{g_{0}} .
$$

Since $G_{p}$ is concave and homogeneous one, we have

$$
G_{p}\left(A^{w}-A^{v}\right) \leq G_{p}\left(A^{w}\right)-G_{p}\left(A^{v}\right)=e^{-2 w}-e^{-2 v} \leq 0 .
$$

Let $\tilde{w}=w-v$. Now we can write $G_{p}\left(A^{w}-A^{v}\right)$ as follows

$$
G_{p}\left(A^{w}-A^{v}\right)=a_{i j}(x) \tilde{w}_{i j}+b_{i}(x) \tilde{w}_{i}
$$

with $\lambda_{0} I d \leq\left(a_{i j}(x)\right) \leq \Lambda_{0} I d$ and $b_{i}(x)$ bounded for any $i$. Therefore, we can apply Theorem 5 on page 61 and Theorem 7 on page 65 in 32 to prove the Lemma.

Remark 4. Proposition 4 does not hold for $p=n-1$. For example $u=k x_{1}$ for any $k>0$ is a solution of

$$
F(u)=G_{n-1}\left(\nabla^{2} u+d u \otimes d u-\frac{|\nabla u|^{2}}{2} g_{0}+S_{g_{0}}\right)=0 .
$$

The same example indicates that without the concavity of $F$, Proposition 1 and Theorem 5 are not true. It is easy to check that $F\left(u_{k}\right)=0$. On a domain $\Omega \subset\left\{x_{1} \geq 0\right\}$, we have $u_{k} \geq 0$. But $\left|\nabla u_{k}\right|=k \rightarrow \infty$

Proposition 5. Let $\left(M, g_{0}\right)$ be a compact Riemannian manifold with Ric $g_{0} \geq(n-1) g_{0}$. If $V_{\max }\left(M,\left[g_{0}\right]\right)=\operatorname{vol}\left(\mathbb{S}^{n}\right)$, then $(M, g)$ is conformally equivalent to the standard unit sphere.

This Proposition is a direct consequence of the following

Proposition 6. Let $(M, g)$ be a compact Riemannian manifold with Ric $_{g} \geq n-1$. If $\operatorname{vol}(M)$ is close to $\omega_{n}$, the volume of $\mathbb{S}^{n}$, then, the Yamabe constant of $(M,[g]), Y(M,[g])$ is close to $n(n-1) \omega_{n}^{2 / n}$, the Yamabe constant of $\mathbb{S}^{n}$.

Proof. Let us first recall the well-known Yamabe constant of $(M, g)$, which is defined by

$$
Y(M,[g]):=\inf \left(\int v^{\frac{2 n}{n-2}} d \operatorname{vol}(g)\right)^{-\frac{n-2}{n}}\left\{4 \frac{n-1}{n-2} \int|\nabla v|^{2} d \operatorname{vol}(g)+\int R_{g} v^{2} d v o l(g)\right\}
$$

By a result of Ilias [20, which is based on a result of Gromov (see also [3]), we have

$$
n(n-1) \omega_{n}^{2 / n}\left(\int v^{\frac{2 n}{n-2}}\right)^{(n-2) / 2} \leq\left(\frac{\omega_{n}}{\operatorname{vol}(g)}\right)^{2 / n}\left\{4 \frac{n-1}{n-2} \int|\nabla v|^{2}+n(n-1) \int v^{2}\right\},
$$

for any $v \in H_{1}^{2}(M)$. Note that $R_{g} \geq n(n-1)$. Therefore, we have

$$
Y(M,[g]) \geq\left(\frac{\omega_{n}}{\operatorname{vol}(g)}\right)^{-2 / n} n(n-1) \omega_{n}^{2 / n} \geq n(n-1) \omega_{n}^{2 / n}-\delta,
$$


for any small $\delta>0$, provided that $\operatorname{vol}(g)$ is close to $\omega_{n}$.

By the results of Colding (see [8, [9] and 31]), we know that the condition in Proposition 6] is equivalent to the one of following three other conditions:

1) $\operatorname{rad} M$ is close to $\pi$,

2) $M$ is Gromov-Hausdorff close to $\mathbb{S}^{n}$,

3) the $(n+1)$ th eigenvalue of the Laplacian, $\lambda_{n+1}(M)$, is close to $n$.

Proof of Proposition 5. $V_{\max }\left(M,\left[g_{0}\right]\right)=\operatorname{vol}\left(\mathbb{S}^{n}\right)$ implies by definition that there is a sequence $g_{i} \in\left[g_{0}\right]_{+}$with $\lim _{i \rightarrow \infty} \operatorname{vol}\left(g_{i}\right)=\operatorname{vol}\left(\mathbb{S}^{n}\right)$. Proposition [6 implies that $Y\left(M,\left[g_{0}\right]\right)=Y\left(M,\left[g_{i}\right]\right) \rightarrow$ $n(n-1) \omega_{n}^{2 / n}$, the Yamabe constant of $\mathbb{S}^{n}$. Hence, the Yamabe constant of $\left(M,\left[g_{0}\right]\right)$ equals to the Yamabe constant of the standard sphere. By the resolution of the Yamabe problem by Aubin [1] and Schoen [37], $\left(M,\left[g_{0}\right]\right)$ is conformally equivalent to the standard sphere.

Proof of Theorem [2. Theorem 2 follows from Propositions 5 and 3.

Remark 5. It is interesting to know weather $V_{\max }$ is achieved as in the negative case. One can show that if $V_{\max }$ is achieved by $\tilde{g}$, then $\mathcal{R}_{\tilde{g}}^{s}$ is constant.

Now we prove Theorem 3 .

Proof of Theorem 3. It follows the exact same arguments in the proof of Theorem 3 in [14, since that proof works for general uniform elliptic concave equations as well, as we note that $W_{p}(g)>0$ implies the positivity of the mean curvature when $p \leq \frac{n}{2}$. We only give a sketch here.

Step 1. We define a deformation

$$
f_{t}(g):=t W_{p}(g)+(1-t) R_{g}=1
$$

where $g=e^{-2 u} g_{0}$. Equation (55) $(\forall t \in[0,1])$ is still uniformly elliptic and concave.

Step 2. (Harnack inequality) There is a constant $C>0$ such that for a solution $u$ of (55) in $B_{3 R}$ we have

$$
\min _{B_{R}} u+\max _{B_{2 R}} u \geq 2 \log R-\log C .
$$

Here $B_{R}$ is the ball of radius $R$ in $\mathbb{R}^{n}$. (56) can be proved as in 14] using the method of moving planes.

By scaling argument, we may assume that $R=1$. Assume by contradiction that (56) is not true. Then there exists a sequence of solutions of (55) in $B_{3}$ such that

$$
\min _{B_{1}} u_{i}+\max _{B_{2}} u_{i}<-i \text {. }
$$

Let $m_{i}=\min _{B_{1}} u_{i}=u_{i}\left(\bar{x}_{i}\right)$, and let $x_{i} \in B_{1}$ with $\bar{B}_{r_{i}}\left(x_{i}\right) \subset \bar{B}_{1}$ and $\left|x_{i}-\bar{x}_{i}\right|=r_{i}$. Here $r_{i}=e^{m_{i}}$. In view of (57), we know that $r_{i} \rightarrow 0$ as $i \rightarrow \infty$. Consider a new sequence of functions $v_{i}$ defined by

$$
v_{i}(x)=u_{i}\left(x_{i}+r_{i} x\right)-m_{i}
$$


and set $\bar{x}_{i}=x_{i}+r_{i} \bar{y}_{i}$. It is clear that $v_{i}$ satisfies (55) in $\left\{|x|<r_{i}^{-1}\right\}$ and $\left|\bar{y}_{i}\right|=1$. ¿From (57), we extend $v_{i}$ by the Kelvin transformation to the whole Euclidean space as in (2.8) of [14. Now applying the method of moving planes as in [14, which in turn follows closely from [7, we can show that $v_{i}$ converges to 0 in $B_{\frac{1}{2}}\left(\bar{y}_{i}\right)$. This is a contradiction. Note that though we are dealing with the Lipschitz operators, the method of moving planes works by using the fact that $f_{t}$ in (55) is uniformly elliptic and concave.

Step 3. Consider a solution $u$ of equation (55). First, it is clear that we have that the scalar curvature of $g=e^{-2 u} g_{0}$ is positive. Hence we can apply the result of Schoen-Yau in 38, to embed the universal cover $\widetilde{M}$ of $(M, g)$ into $\mathbb{S}^{n}$ by a map $\Phi$ conformally. Therefore we can use the method of moving planes (again make use of uniformly ellipticity and concavity of $f_{t}$ ) to obtain as in 14 that

$$
|\nabla u|(x) \leq C, \quad \text { for any } x \in M,
$$

for some constant independent of $u$, provided that $\left(M, g_{0}\right)$ is not equivalent to $\mathbb{S}^{n}$. It follows that

$$
\max u-\min u \leq C,
$$

for some constant independent of $u$. (58), together with the Harnack inequality (56), implies that

$$
\min u \geq C,
$$

for some constant independent of $u$. Hence by Theorem [ we know that the solution space of equation (55) is compact.

Step 4. From Step 3 we can apply the degree theory. We may use a result of Li in [25], a variation of the original Leray-Schauder theorem [24. We also refer to Nirenberg's lecture notes [29] on the exposition of the degree theory in nonlinear differential equations. When $t=0$, the topological degree for equation (55) is -1 , which was proved by Schoen 35. Since the solution space is compact, the topological degree for equation (55) with $t=1$ is also -1 . This finishes the proof of the Theorem.

Remark 6. As in Remark 1, the conditions in Theorem 2 and Theorem 3 can be weakened to the assumption that the corresponding curvature of the background metric is nonnegative and positive at some point. The same argument using the short time existence of the corresponding curvature flows as in Remark 1 can produce a metric $g \in\left[g_{0}\right]$ with the positive curvatures.

\section{ApPEndix}

In this Appendix, we assume that $(M, g)$ is a compact locally conformally flat manifold. Recall the Weitzenböck formula for $p$-forms $\omega$

$$
\Delta \omega=\nabla^{*} \nabla \omega+\mathcal{R} \omega
$$

where

$$
\mathcal{R} \omega=\sum_{j, l=1} \omega_{j} \wedge i\left(e_{l}\right) R\left(e_{j}, e_{l}\right) \omega
$$


Here $e_{j}$ is a local basis and $i(\cdot)$ denotes the interior product $\Delta=d d^{*}+d^{*} d$ is the Hodge-de Rham Laplacian and $\nabla^{*} \nabla$ is the (positive) Laplacian. In local coordinates, let $\omega=\omega_{1} \wedge \cdots \wedge \omega_{p}$. Since $(M, g)$ is locally conformally flat, we have

$$
\mathcal{R} \omega=\left((n-p) \sum_{i=1}^{p} \lambda_{i}+p \sum_{i=p+1}^{n} \lambda_{i}\right) \omega,
$$

where $\lambda$ 's are eigenvalues of the Schouten tenser $S_{g}$. See for instance [28] or [14. By the Bochner technique, we have

Proposition 7. Let $(M, g)$ is a compact locally conformally flat manifold and $1 \leq p \leq n / 2$. If its $p$-Weitzenböck curvature $W_{p}(g)$ is positive, then the Betti number $b_{q}=0$ for $p \leq q \leq n-q$. If its $p$-Weitzenböck curvature $W_{p}(g)$ is non-negative and $b_{p} \neq 0$, then $M$ is a quotient of $H^{p} \times \mathbb{S}^{n-p}$ if $p>1$ and a quotient of $\mathbb{S}^{1} \times \mathbb{S}^{n-1}$ if $p=1$, where $H^{p}$ is the hyperbolic space of curvature -1 and $\mathbb{S}^{n-p}$ is the standard sphere of curvature 1 .

For the vanishing of cohomology groups under various conditions, see [28] and [14] and referrences therein.

\section{REFERENCES}

[1] T. Aubin, Équations différentilles non linéaires et probléme de Yamabe concernant la courbure scalaire, J. Math. Pures Appl., 55 (1976), 269-296.

[2] B. L. Bishop and R. J. Crittenden, Geometry of manifolds, Academic Press, New York, 1964.

[3] G. Besson, G. Courtois and S. Gallot, Volume et entropie minimale des espaces localement symétriques, Invent. Math., 103 (1991), 417-445.

[4] G. Besson, G. Courtois and S. Gallot, Entropies et rigidités des espaces localement symétriques de courbure strictement négative, Geom. Funct. Anal., 5 (1995), 731-799.

[5] L.A. Caffarelli, Interior a priori estimates for solutions of fully nonlinear equations. Ann. of Math., (2) 130 (1989), no. 1, 189-213.

[6] L.A. Caffarelli and Xavier Cabré, Fully nonlinear elliptic equations, Amer. Math. Soc. Colloq. Publ., 43, Amer. Math. Soc., Providence, RI, 1995.

[7] C.C. Chen and C.S. Lin, Estimates of the conformal scalar curvature equation via the method of moving planes, Comm. Pure Appl. Math. 50 (1997), 971-1017.

[8] T. Colding, Shape of manifolds with positive Ricci curvature, Invent. Math., 124 (1996), 175-191.

[9] T. Colding, Large manifolds with positive Ricci curvature, Invent. Math., 124 (1996), 193-214.

[10] L.C. Evans, Classical solutions of fully nonlinear, convex, second-order elliptic equations, Comm. Pure Appl. Math., 35 (1982), 333-363.

[11] L. Gao and S.T. Yau, The existence of negatively Ricci curved metrics on three-manifold. Invent. Math. 85 (1986), 637-652.

[12] M. Gromov, Volume and bounded cohomology, Publ. Math. I.H.E.S., 56 (1981) 213-307.

[13] M. Gromov, Metric structures for Riemannian and non-Riemannian spaces, Progress in Math., 152 Birkhäuser Boston, Inc., Boston, MA, 1999.

[14] P. Guan, C.-S. Lin and G. Wang, Application of the method of moving planes to conformally invariant equations, Math. Z. 247 (2004), 1-19.

[15] P. Guan and G. Wang, Local estimates for a class of fully nonlinear equations arising from conformal geometry, Int. Math. Res. Not., 2003, no. 26 (2003), 1413-1432.

[16] P. Guan and G. Wang, A fully nonlinear conformal flow on locally conformally flat manifolds, J. reine angew. Math., 557 (2003), 219-238. 
[17] P. Guan and G. Wang, Geometric inequalities on locally conformally flat manifolds, Duke Math. Journal, 124, (2004), 177-212.

[18] M. Gursky and J. Viaclovsky, Volume comparison and the $\sigma_{k}$-Yamabe problem. Adv. Math. 187 (2004), $447-487$.

[19] M. Gursky and J. Viaclovsky, Fully nonlinear equations on Riemannian manifolds with negative curvature, Indiana Univ. Math. J., 52 (2003), 399-419.

[20] S. Ilias, Constantes explicites pour les inégalitès de Sobolev sur les variétés riemanniennes compactes. Ann. Inst. Fourier (Grenoble), 33 (1983), no. 2, 151-165.

[21] N.V. Krylov, Boundedly inhomogeneous el domain, Izv. Akad. Nauk SSSR, 47 (1983), 75-108.

[22] N.V. Krylov and M.V. Safonov, An estimate of the probability that a diffusion process hits a set of positive measure, Soviet Math. Dokl., 20 (1979), 253-257.

[23] C. LeBrun, Ricci Curvature, Minimal Volumes, and Seiberg-Witten Theory, Invent. Math. 145 (2001), 279316.

[24] J. Leray and J. Schauder, Topologie et équations fonctionelles, Ann. Sci. École Norm. Sup. 51, (1934), 45-78.

[25] Y. Y. Li, Degree theory for second order nonlinear elliptic operators and its applications, Comm. P. D.E.,14 (1989), 1541-1578.

[26] A. Li and Y. Y. Li, A Liouville type theorem for some conformally invariant fully nonlinear equations, preprint, math.AP/0212376.

[27] J. Lohkamp, Metrics of negative Ricci curvature, Ann. of Math., (2) 140 (1994), 655-683. 1998.

[28] S. Nayatani, Kleinian groups and conformally flat metrics, in: Geometry and global analysis (Sendai, 1993), 341-349.

[29] L. Nirenberg, Topics in nonlinear functional analysis, Lecture Notes, 1973-1974. Courant Institute of Mathematical Sciences, New York University, New York, 1974.

[30] K. Grove and P. Petersen (eds), Comparison geometry, Math. Sci. Res. Inst. Publ., 30, Cambridge Univ. Press, Cambridge, 1997.

[31] P. Petersen, On eigenvalue pinching in positive Ricci curvature, Invent. Math., 138 (1999), 1-21.

[32] M.H. Protter and H.F. Weinberger, Maximum principles in differential equations, Prentice-Hall, Inc., Englewood Cliffs, N.J. 1967.

[33] M.V. Safonov, On the classical solution of Bellman's elliptic equation, Soviet Math. Dokl. 30, (1984), $482-485$.

[34] M.V. Safonov, Classical solution of second-order nonlinear elliptic equations, Math. USSR-Izv., 33 (1989), no. 3, 597-612.

[35] R. Schoen, On the number of constant scalar curvature metrics in a conformal class, Differential Geometry: A symposium in honor of Manfredo Do Carmo (H.B. Lawson and K. Tenenblat, eds), Wiley, 1991, 311-32.

[36] R. Schoen, Analytic aspects of the harmonic map problem, Seminar on nonlinear partial differential equations (Berkeley, Calif., 1983), 321-358, Math. Sci. Res. Inst. Publ., 2, Springer, New York, 1984.

[37] R. Schoen, Conformal deformation of a Riemannian metric to constant curvature, J. Diff. Geom., 20 (1984), 479-495.

[38] R. Schoen and S. T. Yau, Conformally flat manifolds, Kleinian groups and scalar curvature, Invent. Math., 92 (1988), 47-71.

[39] L. Wang, On the regularity of fully nonlinear parabolic equations I $\&$ II, Comm. Pure Appl. Math., 45 (1992), 27-76 \& 141-178.

Department of Mathematics, McGill University, Montreal, H3A 2K6, Canada.

E-mail address: guan@math.mcgill.ca

Max Planck Institute for Mathematics in the Sciences, Inselstr. 22-26, 04103 Leipzig, Germany E-mail address: gwang@mis.mpg.de 